\title{
Acúmulo de mercúrio em sedimentos de fundo da reserva biológica do rio Trombetas (REBIO), cidade de Oriximiná, Brasil
}

O mercúrio $(\mathrm{Hg})$ está presente na Amazônia devido à ocorrência natural e por emissões de garimpo. Os sedimentos de fundo são importantes para estudos ambientais, pois informam sobre o aporte de contaminantes como o Hg nos ambientes aquáticos. Em Porto Trombetas situa-se a área de mineração de bauxita pela Mineradora Rio Norte na área da Floresta Nacional de Saracá-Taquera, próximo a comunidades tradicionais e a REBIO. Assim, considerando a ocorrência de impactos, pretende-se avaliar o acúmulo de $\mathrm{Hg}$ contribuindo para compreender parte da sua dinâmica neste local sem garimpo. Os objetivos são: Avaliar o acúmulo de $\mathrm{Hg}$ em sedimentos de fundo na área da reserva biológica do Rio Trombetas; Quantificar os níveis de Hg nos sedimentos de fundo e caracterizar a qualidade ambiental dos sedimentos de acordo com os níveis de Hg encontrados com o descrito em legislações para áreas não impactadas; Correlacionar os níveis de $\mathrm{Hg}$ nos sedimentos de fundo com a variação sazonal. A área de estudo é a bacia do Rio Trombetas, localizado em Oriximiná, Pará. As amostras de sedimento de fundo superficiais foram coletadas em 30 pontos ao longo da bacia, em quatro amostragens em maio e setembro de 2016 e março e junho de 2017 . As amostras foram guardadas em sacos plásticos, conservadas e levadas para a SAMAM. Após, as amostras foram secas, peneiradas e submetidas à abertura ácida e analisadas com Espectrômetro de Absorção Atômica com Sistema de Vapor Frio. Os resultados mostram que não ocorreu variação sazonal estatística significativa nas concentrações de $\mathrm{Hg}$ Total ( $\mathrm{HgT}$ ), mas ocorreu variação estatística significativ espacial. A variação espacial pode ser explicada nos pontos com maiores concentrações por coincidir justamente nos locais em que se concentram as atividades de mineração de bauxita. De acordo com os valores de TEL e PEL e a resolução do CONAMA no 344/2004 todos os pontos apresentaram concentrações abaixo do nivel 02(PEL) quando é pouco provável ocorrer efeitos adversos na biota. Alguns pontos apresentaram concentracõos médias acima da faixa para rios amazônicos não contaminados. As maiorias dos pontos enquadram-se nos valores regionais amazônicos, com exceções superando os valores regionais. A qualidade ambiental dos sedimentos apresentou-se dentro dos padrões regulares quando comparados com valores de referência internacional e da legislação brasileira. Conclui-se que as atividades como a mineração de bauxita podem contribuir para remobilização do $\mathrm{Hg}$ para os ambientes aquáticos.

Palavras-chave: Mercúrio total; Sedimento; Acúmulo; Mineração.

\section{Mercury accumulation in bottom sediments on Trombetas river biological reserve (REBIO), Oriximiná city, Brazil}

In the Amazon, mercury ( $\mathrm{Hg}$ ) occurs naturally and by gold mining emissions. Bottom sediments are important for environmental investigations as they provide information about the entry of $\mathrm{Hg}$ into the aquatic ecosystem. In Porto Trombetas is situated the bauxite mining area by Mineradora Rio Norte in the Saracá-Taquera National Forest area, near traditional communities and REBIO. Thus, considering the occurrence of impacts, it is intended to evaluate the accumulation of $\mathrm{Hg}$ contributing to understand part of its dynamics in this place without gold mining. The objectives are: to evaluate the accumulation of $\mathrm{Hg}$ in bottom sediments in the area of the Trombetas River biological reserve; quantify $\mathrm{Hg}$ levels in bottom sediment and characterize the environmental quality of sediments according to the $\mathrm{Hg}$ levels found with those described in legislation for non-impacted areas: Correlate the levels of $\mathrm{Hg}$ in bottom sediments with seasonal variation. The study area is the Trombetas River basin, located in Oriximiná, Pará, Brazil. Surface bottom sediment samples were collected at 30 points across the basin, in four samplings in May and September 2016 and March and June 2017. The samples were stored in plastic bags, preserved and taken to SAMAM. Afterwards, the samples were dried, sieved and subjected to acid opening and analyzed with Cold Vapor Atomic Absorption Spectrometer. Results show statistically non-significant seasonal variation in Total $\mathrm{Hg}$ (HgT) concentrations, but it was statistically significant spatial variation. Spatial variation can be explained at the highest concentration points by coinciding precisely where the bauxite mining activities are concentrated. According to the TEL and PEL values and CONAMA Resolution No. 344/2004 all points presented concentrations below level 02 (PEL), when adverse effects on the biota are almost unlikely to occur. Some points presented average concentrations above the range for uncontaminated Amazon rivers. Most of the points fit the Amazonian regional values, with exceptions exce as bauxite mining can contribute to $\mathrm{Hg}$ remobilization to aquatic environments.

Keywords: Total mercury; Sediment; Accumulation; Mining.

Topic: Engenharia Ambiental

Reviewed anonymously in the process of blind peer
Received: 15/04/2021

Approved: 16/05/2021
Francisco Áureo Noronha Filho

Universidade Federal Rural da Amazônia, Brasil

http://lattes.cnpq.br/1168719632959128

fco.aureo.n.f@gmail.com

Thaís Karolina Lisboa de Queiroz (it)

Universidade Federal do Rio de Janeiro, Brasil

http://lattes.cnpq.br/6104005784735107

http://orcid.org/0000-0002-6792-5986

thaiskarolina@hotmail.com

Diomar Oliveira Cavalcante

Universidade Federal do Pará, Brasil

http://lattes.cnpq.br/1801417625863091

diomarcavalcante@yahoo.com.br

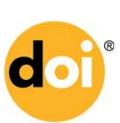

DOI: 10.6008/CBPC2179-6858.2021.005.0031
João Paulo Pereira Góes

Universidade Federal do Rio de Janeiro, Brasil

http://lattes.cnpq.br/0264134579594335

joaopereira@iec.gov.br

Marcelo De Oliveira Lima (iD

Universidade Federal do Pará, Brasil

http://lattes.cnpq.br/2839100072211095

http://orcid.org/0000-0003-3983-1672

marcelolima622@gmail.com
Referencing this:

NORONHA FILHO, F. A.; QUEIROZ, T. K. L.; CAVALCANTE, D. O.; GÓES, J. P. P.; LIMA, M. O.. Acúmulo de mercúrio em sedimentos de fundo da reserva biológica do rio Trombetas (REBIO), cidade de Oriximiná, Brasil. Revista Ibero Americana de Ciências Ambientais, v.12, n.5 p.378-386, 2021. DOI: http://doi.org/10.6008/CBPC21796858.2021.005.0031 


\section{INTRODUÇÃO}

O elemento químico mercúrio $(\mathrm{Hg})$ está presente no ambiente amazônico devido seu ciclo natural, estando presente no ar, água, solo, sedimentos, nos reinos animais e vegetais e chegando ao homem através da cadeia alimentar (TUZEN et al., 2009). Este, desde muito tempo, também pode ser disponibilizado e remobilizado para o ambiente a partir de diversas atividades humanas, como, processos industriais, garimpos artesanais de ouro, construção de hidrelétricas, derrubada de floresta, queimadas, entre outros (HACON et al., 2008).

Os baixos rendimentos dos processos de amalgamação tornam a liberação de $\mathrm{Hg}$ fazem com que a mineração artesanal de ouro seja ainda hoje a principal fonte de emissão antrópicas desse contaminante na Amazônia (LECHLER et al., 2000). Estudos realizados a partir da corrida do ouro na década de 80 nos garimpos dos rios Madeira (RO), Tapajós e Xingu (PA), Parauari (AM) e Jari (AP) mostraram níveis altos de $\mathrm{Hg}$ no ambiente e humanos (MARINHO et al., 2014). No entanto, apesar das atividades mineradoras terem sua localização específica, o Hg liberado nesse processo pode circular por quilômetros, pois o $\mathrm{Hg}$ possui um tempo de trânsito na atmosfera de aproximadamente 2 anos, ou seja, aréas onde não existem relatos de atividades garimpeiras ou até áreas preservadas e pristinas podem registrar elevado background de $\mathrm{Hg}$ influenciado pela deposição de longas distâncias. (PACYNA et al., 2010; FU et al., 2010).

O Hg no ambiente pode ser encontrado em três formas, mercúrio elementar ou vapor de mercúrio $\left(\mathrm{Hg}^{0}\right)$, mercúrio ionizado $\left(\mathrm{Hg}^{+}\right.$e $\left.\mathrm{Hg}^{2+}\right)$ e no compartimento aquático, em condições ideais ( $\mathrm{pH}$ baixo, anaeróbio, presença de matéria orgância, substrato para bactérias sulforedutoras), estas formas podem sofrer metilação originando o composto metilmercúrio $(\mathrm{MeHg})$, substância cujo potencial neurotóxico foi reconhecido após graves acidentes ambientais, como o ocorrido em Minamata no Japão. Uma vez que, este composto tenha sido formado ele é introduzido na cadeia trófica através do processo de biomagnificação podendo causar danos aos organismos aquáticos e a saúde humana (DIRINGER et al., 2014).

Os sedimentos são ambientes propícios para o acúmulo de $\mathrm{Hg}$, pois atuam como reservatórios desse contaminante. Portanto, este compartimento dos ecossistemas aquáticos é o principal depósito de $\mathrm{Hg}$ e funciona como importante fonte deste elemento para os organismos aquáticos. Por esta razão, os sedimentos são considerados uma matriz importante para estudos ambientais e bons indicadores da saúde dos ecossistemas aquáticos, podendo ser empregados na obtenção de uma série de informações associadas ao aporte de $\mathrm{Hg}$ e outras substâncias tóxicas (HORTELLANI et al., 2008).

Nas últimas décadas, a partir de políticas estatais que induziram investimentos internacionais e nacionais, surgiram, na Amazônia, algumas aglomerações minerárias e industriais. Em 1978, foi instalada no território da Cidade de Oriximiná a Mineração Rio do Norte (MRN) para exploração e extração de bauxita nas margens do Rio Trombetas. Esta foi idealizada para produzir a matéria-prima para a produção da alumina e assim integrar a Cadeia Produtiva do Alumínio no Estado do Pará (ALUNORTE'). A área de extração mineral foi estabelecida em área de mata fechada circunvizinha de terras quilombolas e ao lado da Reserva Biológica do Rio Trombetas (REBIO Trombetas). Na margem direita do rio Trombetas foi

\footnotetext{
${ }^{1}$ http://www.alunorte.net
} 
construído um porto que passou a integrar o distrito de Porto Trombetas. Este porto foi idealizado para exportação de grande quantidade de minério bruto seco, sendo o escoamento favorecido pela boa profundidade da calha do rio trombetas e, desta forma, apresentar excelente navegabilidade para navios de grande porte (SODRÉ et al., 2015).

A lama vermelha, resíduo proveniente do beneficiamento do minério de bauxita é composta de óxidos de ferro, sílica, alumina e outros metais pesados, para cada tonelada de alumina produzida a quantidade de lama vermelha produzida varia de 0,3 a 2,5 toneladas, a depender das características da bauxita (TUAZON et al., 2008). Quando os resíduos de mineração são expostos à água e condições oxidantes podem ocorrer a produção de drenagem de mina ácida. Segundo Kusin et al. (2018), esse tipo de drenagem contém altas concentrações de metais potencialmente tóxicos, dentre estes o Hg. Esta atividade de extração mineral gera grandes quantidades de resíduos sólidos que normalmente são acumulados em bacias de decantação, apesar de existir registros desse acúmulo em lagos da região do Trombetas, como o lago do Batata. Os efluentes líquidos desses processos são despejados diretamente no leito dos rios e lagos da região. Tanto esses resíduos sólidos como estes efluentes carreiam também metais potencialmente tóxicos, como o $\mathrm{Hg}$, que se encontravam imobilizados na rocha e passam a ser disponibilizados para o ambiente (KUSIN et al., 2017; ABDULLAH et al., 2016).

Em virtude da presença desse complexo minerário em proximidade a comunidades tradicionais, a REBIO Trombetas, e a Floresta Nacional (FLONA) de Saracá-Taquera, e considerando que nos seus resíduos sólidos e efluentes são também liberados metais potencialmente tóxicos, como o $\mathrm{Hg}$, nesta proposta pretende-se avaliar o acúmulo de mercúrio nos sedimentos de fundo de parte da bacia do Rio Trombetas entre a FLONA e a REBIO. De forma que conhecer acerca deste acúmulo possibilita ainda a compreensão da dinâmica do mercúrio na região que não possui atividades de extração de ouro.

\section{METODOLOGIA}

A área de estudo constitui a bacia do Rio Trombetas, um dos principais tributários da margem direita do rio Amazonas, e quase toda sua extensão está localizada no Município de Oriximiná, noroeste do estado do Pará. O rio nasce fora da área de preservação ambiental com sua origem no escudo das Guianas. A partir do distrito de Cachoeira Porteira o Rio Trombetas tem como sua margem direita a Reserva Biológica do Rio Trombetas (REBIO Trombetas) e em sua margem esquerda Terras quilombolas do Alto-Trombetas e o distrito de Porto trombetas, onde está localizada a Sede da Mineradora Rio do Norte (MRN), uma das maiores exploradoras de bauxita no mundo.

O rio trombetas avança tendo como margem a REBIO até as terras quilombolas do Erepecuru delimitadas pelo rio Cuminá, mais a leste de Oriximiná. Na sua extensão também são observadas, mesmo que esporadicamente, algumas comunidades ribeirinhas (quilombolas) que utilizam o rio para atividades como a pesca de subsistência, ver na Figura 01.

As amostras de sedimento de fundo superficiais foram coletadas em pontos definidos ao longo do rio Trombetas, Figura 01, realizados em quatro amostragens nos meses de maio e setembro de 2016 e março e 
junho de 2017, foram selecionados 30 pontos de amostragem de sedimento ao longo da bacia do Rio Trombetas sob influência da REBIO. Nestas atividades a coleta realizada com o auxílio de um amostrador de sedimento de fundo Van-Ven. Foram amostrados os sedimentos de fundo em profundidades variadas que após serem retirados do amostrador foram transferidos para sacos plásticos Zip Lock. Posteriormente no laboratório, as amostras foram conservadas em freezer à temperatura $-4^{\circ} \mathrm{C}$. Todos os pontos amostrados foram georreferenciados mediante o uso de um posicionador do tipo global positioning system (GPS).

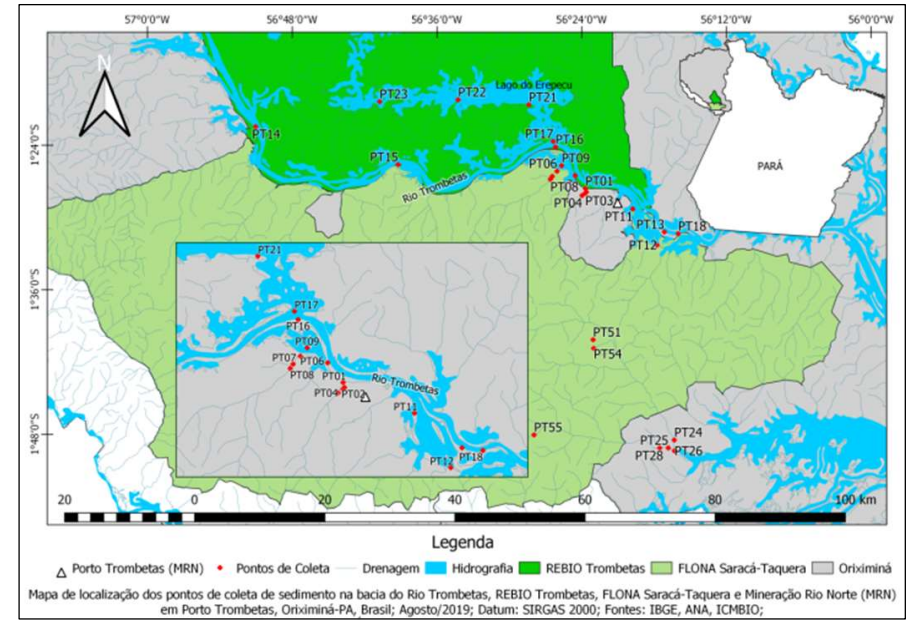

Figura 1: Mapa de localização da dos pontos de coleta de sedimento de fundo na bacia do Rio Trombetas, próximo a FLONA Saracá-Taquera, a REBIO Trombetas e ao distrito de Porto Trombetas.

O pré-tratamento das amostras foi realizada na sala de solos da Seção de Meio Ambiente (SAMAM). De tal forma as amostras de sedimento foram despejadas sobre vasilhas e expostas a temperatura ambiente até secar totalmente, método de terra fina seca ao ar (TFSA). Posteriormente foram homogeneizadas utilizando-se grau de ágata, e por fim, realizou-se a separação com a peneira granulométrica, para obter partículas menores que 270 mesh, a fração fina. A abertura ácida procedeu pela pesagem de 100-200 mg do sedimento (30 \% das amostras feitas em replicata), adição de $1 \mathrm{~mL}$ de água deionizada, $2 \mathrm{~mL}$ de $\mathrm{HNO}_{4}$ : $\mathrm{HClO}_{4}$ (1:1) e $5 \mathrm{~mL}$ de $\mathrm{H}_{2} \mathrm{SO}_{4}$, seguida do aquecimento em chapa aquecedora a 200-230C C, durante 30 minutos. Após resfriamento em temperatura ambiente, a solução foi aferida para o volume final de $50 \mathrm{~mL}$ com água deionizada. A análise de $\mathrm{HgT}$ foi realizada seguindo metodologia de Akagi, et al. (2004), utilizando Espectrômetro de Absorção Atômica com Sistema de Vapor Frio (CV-AAS), em Mercury Analyzer (SANSO), modelo Hg-201.

Para tratamento estatístico dos dados foram utilizados os softwares Bioestat 5.3, PAleontological STastistis (PAST 3.25) e Microsoft Excel 2010. Este último foi utilizado na organização/tabulação dos dados e elaboração de gráficos para apresentar os resultados. Quanto ao uso dos outros dois softwares segue a descrição adiante. O primeiro procedimento foi verificar a normalidade dos dados conforme o teste de Shapiro-Wilk $(\alpha=0,05)$. Após o teste de normalidade os dados que não apresentaram distribuição gaussiana foram submetidos ao teste de Kruskal-Wallis $(\alpha=0,05)$ para analisar a variação entre os meses (variação sazonal) e entre os pontos (variação espacial). Foi realizada análise de cluster (AC) com o objetivo de avaliar os possíveis acúmulos nos pontos de coletas ao longo do período de estudo, a fim de associá-los à proximidade das atividades minerarias na região. 


\section{RESULTADOS E DISCUSSÃO}

As análises das 82 amostras relativas aos 30 pontos foram realizadas no período de agosto de 2018 a abril de 2019. Assim a concentração de HgT nas amostras de sedimento de cada ponto está representadas na Figura 02 de acordo com a campanha de coleta. Uma vez que o número de amostras e pontos das campanhas diferem entre si, há pontos que apresentam resultados de apenas uma das campanhas. Pois não foi possível realizar a coleta em alguns pontos em todas as campanhas, em virtude da elevada profundidade temporária dos corpos hídricos locais (elevação do nível do rio em função dos pulsos de inundação), assim impedindo o amostrador de sedimento alcançar o fundo e obter amostra em quantidade suficiente.

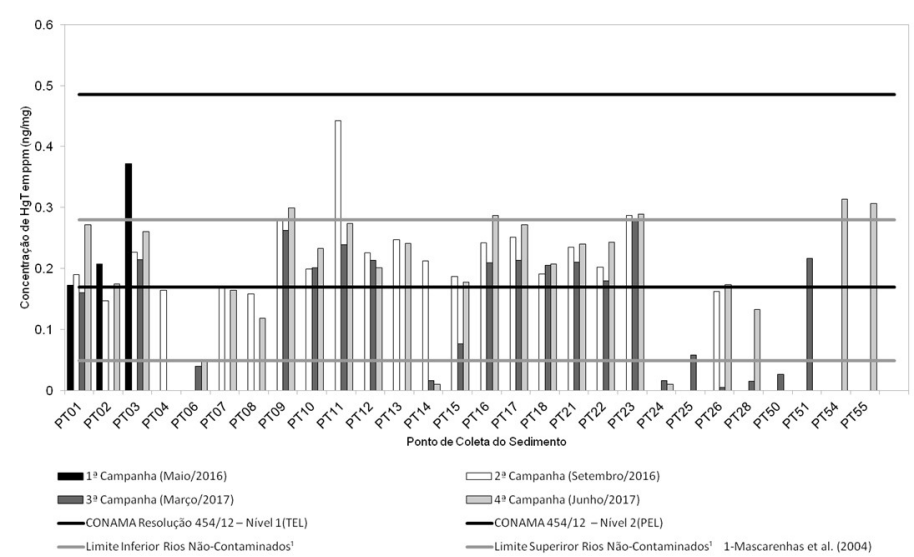

Figura 02: Concentração de mercúrio total nas amostras de sedimento por ponto e campanha/mês de coleta.

Ao analisar os resultados agrupados por campanha, Tabela 1, nos quatro meses, concluiu-se que não houve variação estatística significativa entre as concentrações de HgT nas amostras de sedimentos pelo teste de Kruskal-Wallis $(\mathrm{H}=5,315, \mathrm{p}=0,1501)$.

Tabela 1: Concentrações médias de $\mathrm{HgT}$ (ng. $\mathrm{mg}^{-1}$ ) nas amostras de sedimento de acordo com a campanha (mês/ano).

\begin{tabular}{cccccccc}
\hline Campanha (Mês/Ano) & Média & \pm & DP & Mediana & N & Máximo & Mínimo \\
\hline 1a (maio/2016) & 0.2503 & \pm & 0.1064 & 0.2071 & 03 & 0.3714 & 0.1722 \\
\hline 2a (setembro/2016) & 0.2210 & \pm & 0.0653 & 0.2076 & 20 & 0.4421 & 0.1475 \\
\hline 3a (março/2017) & 0.1457 & \pm & 0.0958 & 0.2010 & 21 & 0.2797 & 0.0054 \\
\hline 4a (junho/2017) & 0.2064 & \pm & 0.0892 & 0.2362 & 24 & 0.3140 & 0.0105 \\
\hline
\end{tabular}

DP: Desvio Padrão; N: número de amostras; „: mais ou menos;

Após analisar os resultados agrupados por ponto, Tabela 2, pode ser observado que houve variação estatística significativa entre as concentrações de $\mathrm{HgT}$ nas amostras de sedimento, através do Teste de Kruskal-Wallis, $\mathrm{H}=56,13, \mathrm{p}=0,0008279$. Logo, a concentração de $\mathrm{HgT}$ teve variação espacial.

$\mathrm{Na}$, na Figura 03, está representado o resultado da Análise de Cluster realizada apenas com os pontos que apresentaram os dados pareados para as três últimas campanhas, onde o eixo Y representa a distância entre os pontos que, calculada levando em consideração a concentração de $\mathrm{HgT}$ em cada mês/campanha de cada ponto.

Os resultados aqui encontrados sugerem que não ocorreu variação sazonal estatisticamente 
significativa nas concentrações de HgT. Logo se sugere também que os períodos de cheia e seca no rio Trombetas não influenciam na variação da concentração de $\mathrm{HgT}$ no sedimento de fundo do rio. Este fato pode estar relacionado ao fato de que o Rio Trombetas não sofre influência de maré, sendo observada apenas a cheia e seca do corpo hídrico. Além do mais os pontos representam mais lagos e igarapés de maneira que seria necessário mais pontos para ser mais representativos da calha do Rio Trombetas e o objetivo do trabalho é a caracterização na bacia. Os pontos 03, 09, 11, 16, 23, 54 e 55 apresentaram as concentrações médias mais elevadas de $\mathrm{HgT}$ ou em, pelo menos, um dos meses estudados e estão destacados na Figura 04 assim: "Pontos maior [HgT]". Destes, dois pontos apresentaram as maiores concentrações no período estudado, isto se explica pela proximidade a locais que outrora foram destinados à bacia de rejeitos da mineração de bauxita existente na região conforme foi verificado no local e momento da coleta para os pontos 03 e 11 .

Tabela 2: Concentrações médias de $\mathrm{HgT}$ (ng. $\mathrm{mg}^{-1}$ ) nas amostras de sedimento de acordo com o ponto coletado.

\begin{tabular}{|c|c|c|c|c|c|c|}
\hline Ponto & $\mathbf{N}$ & Média & \pm & DP & Mínimo & Máximo \\
\hline PT01 & 04 & 0.1987 & \pm & 0.0502 & 0.1608 & 0.2719 \\
\hline PT02 & 03 & 0.1764 & \pm & 0.0299 & 0.1475 & 0.2071 \\
\hline PT03 & 04 & 0.2683 & \pm & 0.0715 & 0.2141 & 0.3714 \\
\hline PT04 & 01 & 0.1642 & \pm & - & - & - \\
\hline PT06 & 02 & 0.0448 & \pm & 0.0077 & 0.0394 & 0.0503 \\
\hline PT07 & 02 & 0.1682 & \pm & 0.0052 & 0.1645 & 0.1719 \\
\hline PT08 & 02 & 0.1384 & \pm & 0.0278 & 0.1187 & 0.1580 \\
\hline PT09 & 03 & 0.2798 & \pm & 0.0182 & 0.2629 & 0.2990 \\
\hline PT10 & 03 & 0.2110 & \pm & 0.0189 & 0.1992 & 0.2328 \\
\hline PT11 & 03 & 0.3183 & \pm & 0.1087 & 0.2386 & 0.4421 \\
\hline PT12 & 03 & 0.2132 & \pm & 0.0120 & 0.2014 & 0.2253 \\
\hline PT13 & 02 & 0.2438 & \pm & 0.0042 & 0.2408 & 0.2468 \\
\hline PT14 & 03 & 0.0797 & \pm & 0.1151 & 0.0105 & 0.2126 \\
\hline PT15 & 03 & 0.1473 & \pm & 0.0612 & 0.0768 & 0.1869 \\
\hline PT16 & 03 & 0.2463 & \pm & 0.0389 & 0.2094 & 0.2870 \\
\hline PT17 & 03 & 0.2454 & \pm & 0.0295 & 0.2134 & 0.2713 \\
\hline PT18 & 03 & 0.2015 & \pm & 0.0091 & 0.1911 & 0.2078 \\
\hline PT21 & 03 & 0.2284 & \pm & 0.0153 & 0.2109 & 0.2396 \\
\hline PT22 & 03 & 0.2083 & \pm & 0.0322 & 0.1794 & 0.2430 \\
\hline PT23 & 03 & 0.2853 & \pm & 0.0049 & 0.2797 & 0.2890 \\
\hline PT24 & 02 & 0.0135 & \pm & 0.0042 & 0.0105 & 0.0164 \\
\hline PT25 & 01 & 0.0583 & - & - & - & - \\
\hline PT26 & 03 & 0.1136 & \pm & 0.0939 & 0.0054 & 0.1734 \\
\hline PT28 & 02 & 0.0737 & \pm & 0.0832 & 0.0149 & 0.1325 \\
\hline PT50 & 01 & 0.0265 & \pm & - & - & - \\
\hline PT51 & 01 & 0.2166 & \pm & - & - & - \\
\hline PT54 & 01 & 0.3140 & \pm & - & - & - \\
\hline PT55 & 01 & 0.3068 & \pm & - & - & - \\
\hline
\end{tabular}

A variação espacial da concentração de $\mathrm{HgT}$ representada na Figura 04 pode ser explicada nos pontos com maiores concentrações encontradas justamente nos locais em que se concentram a maioria das atividades de uso e alteração do solo na FLONA de Saracá-Taquera pela Mineradora Rio Norte que são os processos de mineração de bauxita. Estas atividades de uso e alteração podem ser a fonte do mercúrio acumulado nestes pontos, uma vez que não há atividades de extração de ouro e utilização de amalgamação com mercúrio nas vizinhanças imediatas da região (LACERDA, 2008) Além do mais os pontos de maiores acumulações são lagos e igarapés que apresentam grande quantidade de matéria orgânica residual das florestas aliado ao fato de estes ambientes lênticos são mais favoráveis a acúmulo e sedimentação (ALMEIDA, 2014). 




Figura 04: Mapa da distribuição espacial da concentração média de HgT em sedimento de fundo na bacia do Rio Trombetas pelo método geostatístico inverso da potência das distâncias

Em relação ao ponto 11 coletado em setembro de 2016 (2ª campanha), a concentração média foi de 0,4420 ng. $\mathrm{mg}^{-1}$ de HgT, este ponto situa-se no chamado lago do Batata, próximo a antiga bacia de rejeitos. A análise em replicata foi realizada três vezes, pois as duas primeiras replicatas não apresentaram coeficiente de variação dentro do padrão esperado (menor que 20\%) atribuindo a hipótese de que o sedimento coletado não apresentou homogeneidade. A respectiva amostra apresentou coloração avermelhada, em cor característica da lama vermelha residual de bauxita. Esta característica pode também explicar a variação do resultado deste ponto nas duas campanhas seguintes que foram de $0,2386 \mathrm{ng} \cdot \mathrm{mg}^{-1} \mathrm{e}$ 0,2741 ng. $\mathrm{mg}^{-1}$. A análise de Cluster (AC), Figura 03, também permitiu visualizar a singularidade desse ponto que ficou agrupado com a segunda maior distância em relação aos outros pontos e bem separado dos outros agrupamentos.

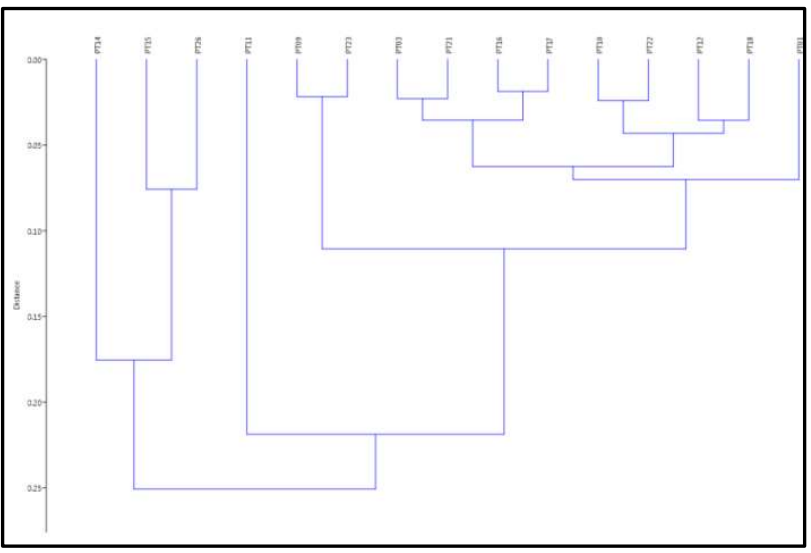

Figura 3: Gráfico do agrupamento dos pontos de acordo com a concentração de HgT por ponto nas campanhas 2, 3 e 4, representado pela distância entre os pontos.

A AC não seguiu um padrão de agrupamento dos pontos com maiores concentrações de acordo com as médias, a semelhança maior foi conforme a comparação das concentrações mensais. Logo através da análise não foi possível encontrar um padrão de acumulação de $\mathrm{Hg}$ no sedimento de fundo.

Embora os pontos 03 e 11 possuam concentrações médias em, pelo menos, um dos meses, enquadradas na qualidade regular de sedimento, de acordo com os valores de TEL e PEL estabelecidos com base nos valores do Canadian Council of Ministers of the Environment (CCME, 2002), todos os outros pontos apresentaram concentração média enquadrada no intervalo de qualidade ótima a boa. Isto 
considerando apenas o contaminante metálico mercúrio. Os mesmos limites do TEL $\left(0,017\right.$ ng. $\left.\mathrm{mg}^{-1}\right)$ e PEL $\left(0,48\right.$ ng. $\left.\mathrm{mg}^{-1}\right)$ são referências para a resolução do CONAMA no 454/2012, sendo respectivamente, nível 1 (limiar abaixo do qual há menor probabilidade de efeitos adversos à biota) e nível 2 (limiar acima do qual há maior probabilidade de efeitos adversos à biota). De tal forma todos os pontos apresentaram concentrações abaixo do nível 2 e acima do nível 1, com exceção do ponto 11 que apresentou concentração próximo ao limiar do nível 2, pouco provável que ocorra efeitos adversos na biota.

Os pontos 03, 09, 11, 16, 23, 54 e 55 apresentaram as maiores concentrações médias de HgT ou em, pelo menos, um dos meses/campanhas estudados acima da faixa para rios amazônicos não contaminados de acordo com Mascarenhas et al. (2004). Sugerindo o fato que a origem do HgT pode ser de fontes geogênicas antrópicas, ou seja, nesse caso a mineração pela atividade de uso e alteração do solo intensifica o acúmulo de mercúrio nos respectivos pontos. Uma vez que, na região amazônica, tais atividades como desmatamento, revolvimento do solo e erosão cujo escoamento ou transporte das partículas de solo estão associados ao aporte de Hg nos ambientes aquáticos (LACERDA, 2008). Visto que ocorre a remobilização do metal imobilizado por adsorção nas frações finas do solo ou rochas e fragmentos de minerais, pode ocorrer também a biodisponibilização do Hg. Estudos realizados nesta mesma área pelo Instituto Evandro Chagas (FAIAL, 2005) peixes com concentrações de HgT, em tecidos de músculo, superiores aos limites recomendados pela Organização Mundial da Saúde e pela Agência Nacional de Vigilância Sanitária para consumo humano. Nos demais pontos as concentrações estão dentro da faixa e em torno do background para rios amazônicos. Logo sugerindo o fato de a origem do HgT ser de fontes geogênicas/naturais. Considerando o estudo de Mascarenhas et al. (2004) que cita o estudo de Pfeiffer et al. (1989). Este autor estabeleceu o background de $0,2 \mathrm{ng} \mathrm{mg}^{-1}$ para rios amazônicos não contaminados por Hg. Além disso, Mascarenhas cita também a faixa estabelecida para rios amazônicos não contaminados $\left(0,05-0,28 \mathrm{ng} \cdot \mathrm{mg}^{-1}\right)$ de Lacerda et al. (1987).

\section{CONCLUSÕES}

Ao final da pesquisa é possível concluir através do estudo que as atividades de uso e alteração do solo e outras atividades que envolvem transporte das partículas de solo, tais como a mineração de bauxita, podem contribuir para remobilização de metais como o $\mathrm{Hg}$ para os ambientes aquáticos, ocasionando acúmulo nos sedimentos de fundo dos rios e lagos. Fator que explica a variação espacial encontrada. A maioria das concentrações de HgT enquadram-se nos valores regionais amazônicos, com poucas exceções que superam os valores regionais.

A qualidade ambiental dos sedimentos apresentou-se dentro dos padrões regulares quando comparados com valores de referência internacional e da legislação brasileira. Não foi possível correlacionar os níveis de mercúrio com os períodos de cheia e seca do Rio Trombetas, uma vez que não foi observada variação sazonal na concentração de HgT nas amostras.

\section{REFERÊNCIAS}


Bauxite Mining in Kuantan. Malays J. Med. Sci.: MJMS, v.23 n.3, p.1-8, 2016.

ALMEIDA, R.. Pulso de inundação e dinâmica espacial do mercúrio em sedimentos no lago Puruzinho, Amazônia Brasileira. Acta Amaz, v.44, n.1, p.99-105, 2014.

BRASIL. Plano de manejo da reserva Biológica do Rio Trombetas. Brasília: Ministério do Meio Ambiente, 2004.

CCME. Canadian Council of Ministers of the Environment. Canadian Sediment Quality Guidelines for the Protection of Aquatic Life. Canadian Environmental Quality Guidelines Summary Tables, 2002.

CONAMA. Conselho Nacional de Meio Ambiente. Resolução N 454. Brasília: CONAMA, 2012.

DIRINGER, S. E.; FIENGOLD, B. J.; ORTIZ, E. J.; GALLIS, J. A.; ARA'UJO-FLORES, J. M.; BERKY, A.; PAN, W. K. Y.; HSU-KIM, H.. River transport of mercury from artisanal and small-scale gold mining and risks for dietary mercury exposure in Madre de Dios, Peru. Environmental Science Processes \& Impacts, v.17 n.2, 2014.

FAIAL, K. R. F.; SANTOS, E. C. O.; BRABO, E. S.; SÁ, G. C.; JESUS, I. R. M.; LIMA, M. O.; MENDES, R. A.; MASCARENHAS, A. F. S.. Mercure levels in fish of Trombetas river in low Amazon área and área out of the influence of gold mining. Cadernos Saúde Coletiva, v.13, n.1, p.237-248, 2005.

FU, X. W.; FENG, X.; DONG, Z. Q.; YIN, R. S.; WANG, J. X.; YANG, Z. R.; ZHANG, H.. Atmospheric gaseous elemental mercury (GEM) concentrations and mercury depositions at a high-altitude mountain peak in south China. Atmospheric Chemistry and Physics, V.10, p.2425-2437, 2010.

HACON, S.; BARROCAS, P. R. G.; VASCONCELLOS, A. C. S.; BARCELLOS, C.; WASSERMAN, J. C; CAMPOS, R. C.; RIBEIRO, C.; AZEVEDO-CARLONI, F. B. An overview of mercury contamination research in the Amazon basin with an emphasis on Brazil. Cadernos de Saúde Pública, v.24, n.7, p.1479-1492, 2008.

HORTELLANI, M. A.; SARKIS J. E. S.; ABESSA D. M. S.; SOUSA E. C. P. M.. Avaliação da contaminação por elementos metálicos dos sedimentos do estuário Santos. Quím. Nova, v.31, n.1, p.10-19, http://dx.doi.org/10.1590/S010040422008000100003.

KUSIN, F. M.; RAHMAN, M. S. A.; MADZIN, Z.. The occurrence and potential ecological risk assessment of bauxite mineimpacted water and sediments in Kuantan, Pahang,Malaysia. Environ. Sci. Pollut, v.24, p.1306-1321, 2017. DOI: https://doi.org/10.1007/s11356-016-7814-7

KUSIN, F. M.; AZANIA, M.; HASANA, S. N. M.; SULONGA, S. N. A.. Distribution of heavy metals and metalloid in surface sediments of heavilymined area for bauxite ore in Pengerang, Malaysia and associated risk assessment. Catena, v.165, p.454-464, 2018.
LACERDA, L. D.; PFEIFFER, W. C.; SILVEIRA, E. G.; BASTOS, W. R.; SOUZA; C. M. M.. Contaminação por mercúrio na Amazônia; análise preliminar do rio Madeira, RO. In: CONGRESSO BRASILEIRO DE GEOQUÍMICA. Anais. Sociedade Brasileira de Geoquímica, 1987.

LACERDA, L. D.; MALM, O.. Contaminação por mercúrio em ecossistemas aquáticos: uma análise das áreas críticas. Estudos Avançados, v.22, n.63, p.173-190, 2008. DOI: http://dx.doi.org/10.1590/S0103-40142008000200011

LECHLER, P. J.; MILLER, J. R.; LACERDA, L. D.; VINSON, D.; BONZONGO, J. C.; LYONS, W. B.; WARWICK, J. J.. Elevated mercury concentrations in soils, sediments, water, and fish of the Madeira River basin, Brazilian Amazon: a function of natural enrichments? The Science of the Total Environment, v.260, p.87-96, 2000.

MARINHO, J.; S.; LIMA, M. O.; SANTOS, E. C. O.; JESUS, I. M.; PINHEIRO, M. C. N.; ALVES, C. N.; MULLER, R. C. S.. Mercury Speciation in Hair of Children in Three Communities of the Amazon, Brazil. Bio Med Research International, v.2014, p.1-9, 2014.

MASCARENHAS, A. F. S.; BRABO, E. S.; SILVA, A. P.; FAIAL, K. F.; JESUS, I. M.; SANTOS, E. C. O.. Avaliação da concentração de mercúrio em sedimentos e material particulado no rio Acre, estado do Acre, Brasil. Acta Amazon, v.34, p.61-80, 2004. DOI: http://dx.doi.org/10.1590/S004459672004000100008

PACYNA, E. G.; PACYNA, J. M.; SUNDSETH, K.; MUNTHE, J.; KINDBOM, K.; WILSON, S.; STEENHUISEN, F.; MAXSON, P.. Global emission of mercury to the atmosphere from anthropogenic sources in 2005 and projections to 2020 . Atmospheric Environment, v.44, p.2487-2499, 2010.

PFEIFFER, W. C.; LACERDA, L. D.; SOUZA, C. M. M.; SILVEIRA, E.; BASTOS, W. R.. Mercury Concentrations in inland waters of gold mining areas in Rondônia, Brazil. The Science of the Total Environment, p.233-240, 1989.

SODRÉ, E. O.; LOPES, P. M.; FIGUEIREDO-BARROS, M. P.; ROLAND, F.; ESTEVES, F. A.; BOZELLI, R. L.. The effects of mining tailings and flood pulse on zooplankton in an Amazonian floodplain environment (Batata Lake, Pará, Brazil). Boletim do Museu Paraense Emílio Goeldi. Ciências Naturais, v.10, n.2, p.247-259, 2015.

TUAZON, D.; CORDER, G. D.. Life cycle assessment of seawater neutralised red mudfor treatment of acid mine drainage. Resources, Conservation and Recycling, v.52, p. 1307-1314, 2008.

TUZEN, M.; KARAMAN, I.; CITAK, D.; SOYLAK, M.. Mercury(II) and methyl mercury determinations in water and fish samples y using solid phase extraction and cold vapouratomic absorption spectrometry combination. Food and Chemical Toxicology, v.47, p.1648-1652, 2009.

A CBPC - Companhia Brasileira de Produção Científica (CNPJ: 11.221.422/0001-03) detém os direitos materiais desta publicação. Os direitos referem-se à publicação do trabalho em qualquer parte do mundo, incluindo os direitos às renovações, expansões e disseminações da contribuição, bem como outros direitos subsidiários. Todos os trabalhos publicados eletronicamente poderão posteriormente ser publicados em coletâneas impressas sob coordenação da Sustenere Publishing, da Companhia Brasileira de Produção Científica e seus parceiros autorizados. Os (as) autores (as) preservam os direitos autorais, mas não têm permissão para a publicação da contribuição em outro meio, impresso ou digital, em português ou em tradução. 